\title{
SPECTRAL MEASURES OF FACTOR OF I.I.D. PROCESSES ON VERTEX-TRANSITIVE GRAPHS
}

\author{
ÁGNES BACKHAUSZ AND BÁLINT VIRÁG
}

\begin{abstract}
We prove that a measure on $[-d, d]$ is the spectral measure of a factor of i.i.d. process on a vertex-transitive infinite graph if and only if it is absolutely continuous with respect to the spectral measure of the graph. Moreover, we show that the set of spectral measures of factor of i.i.d. processes and that of $\bar{d}_{2}$-limits of factor of i.i.d. processes are the same.
\end{abstract}

Keywords: factor of i.i.d.; Gaussian process, spectral measure.

\section{INTRODUCTION}

We consider invariant random processes on vertex transitive graphs that can be performed with "randomized local" algorithms (factor of i.i.d. processes). The aim of the paper is characterizing the covariance structures of factor of i.i.d. processes, in terms of the absolute continuity of their spectral measure with respect to the spectral measure of the graph.

1.1. Factor of i.i.d. processes. Let $G=(V(G), E(G))$ be a vertextransitive graph with countable vertex set $V(G)$. We assign a random variable $X_{v}$ to each $v \in V(G)$. We get an invariant random process if the joint distribution is invariant under the automorphisms of $G$. These are the analogues of stationary processes on $\mathbb{Z}$, and they may be interesting on their own right (Arnaud 1980, 1994).

Among invariant random processes, we deal with factor of i.i.d. processes, see e.g. Elek and Lippner (2010), Hatami, Lovász and Szegedy (2014) or the paper of Lyons (2014) and the references therein. Results of randomized local algorithms (or constant-time parallelized algorithms) belong to this class. Furthermore, factor of i.i.d. processes can be useful for finding large independent sets (Csóka, Gerencsér, Harangi and Virág 2014, Harangi and Virág 2013, Hoppen and Wormald 2013), matchings on nonamenable graphs (Lyons and Nazarov 2011, Csóka and Lippner 2012), colorings, other structures (Gaboriau and Lyons 2009, Kun 2013, Backhausz and Szegedy 2014). This family of processes may also be interesting from an ergodic theoretic point of

Date: 2 September 2016.

2010 Mathematics Subject Classification. 60G15. 
view (Bowen 2010), as they are the factors of the Bernoulli shift. To define factor of i.i.d. processes, loosely speaking, we start with independent and identically distributed labels (from $\mathbb{R}$ ) on the vertices. Then each vertex gets a new label, depending on the labelled rooted graph as it is seen from that vertex. The rule is fixed, and it is the same for all vertices. See Subsection 2.2 for the precise definition.

1.2. Spectral measures, covariance structures and $\bar{d}_{2}$ limit. We assign a finite measure on $\mathbb{R}$ to invariant random processes on $G$, which is the spectral measure of the process. (Throughout the paper, we always consider Borel measures.) This measure is the spectral measure of the graphing associated to the process (Section 2.3). This is also related to the covariance structure of the process, as the following is satisfied for a process $X$ :

$$
\mathbb{E}\left(\left[A^{k} X\right]_{o}, X_{o}\right)=\left\langle A^{k} \delta_{o}, c_{X}\right\rangle_{G}=\int t^{k} d \mu_{X}(t) \quad(k \geq 0),
$$

where $A$ is the adjacency operator, $c_{X}: V \rightarrow \mathbb{R}$ is the covariance structure assigning $\operatorname{cov}\left(X_{o}, X_{v}\right)$ to each vertex $v$, and $\mu_{X}$ is the spectral measure of $X$.

Our goal is characterizing the set of spectral measures of factor of i.i.d. processes. As we will see, the same characterization holds for $\bar{d}_{2}$-limits of factor of i.i.d. processes. The following metric is based on Ornstein's $\bar{d}$-metric (see e.g. Lyons 2014).

Definition 1. The $\bar{d}_{2}$-distance of the random invariant processes $X, X^{\prime}$ (with marginals having finite second moments) is defined as follows.

$$
\begin{array}{r}
\bar{d}_{2}\left(X, X^{\prime}\right)^{2}=\min \left\{\mathbb{E}\left[\left(Y_{o}-Y_{o}^{\prime}\right)^{2}\right]: Y \stackrel{d}{=} X, Y^{\prime} \stackrel{d}{=} X^{\prime},\right. \\
\left.\left(Y, Y^{\prime}\right) \text { is invariant }\right\} .
\end{array}
$$

If the graph is the $d$-regular tree (throughout the paper, we denote by $T_{d}$ the infinite $d$-regular tree), the spectral measure equals to the Fourier transform of the covariance structure, see e.g. Cartier (1973), Arnaud (1980, 1994), Figà-Talamanca and Picardello (1982). On the other hand, in Backhausz, Szegedy and Virág (2015) a uniform exponential bound was proved for the decay of the correlation sequence of a factor of i.i.d. process, and the pointwise closure of the possible correlation sequences was described in the case $G=T_{d}(d \geq 3)$.

1.3. Main results. Our main result is the following theorem, which characterizes the spectral measures of factor of i.i.d. processes, linear factor of i.i.d. processes (see Definition 13 and [14) and $\bar{d}_{2}$-limits of factor of i.i.d. processes (Definition 1). 
Theorem 2. Fix an infinite vertex-transitive graph $G$. Suppose that all processes below have marginals with mean 0 and finite second moment. The following are equivalent for a finite (Borel) measure on $\mathbb{R}$.

(i) It is absolutely continuous with respect to the spectral measure $\nu$ of the graph $G$.

(ii) It is the spectral measure of some linear factor of i.i.d. process.

(iii) It is the spectral measure of some factor of i.i.d. process.

(iv) It is the spectral measure of some limit of factor of i.i.d. process with respect to the $\bar{d}_{2}$-distance.

$(v)$ It is the spectral measure of some invariant process which is the $\bar{d}_{2}$-limit of processes with spectral measures satisfying $(i)$.

The equivalence of $(i i i)$ and $(i v)$ shows that the conjecture about $\bar{d}_{2^{-}}$ limits of factor of i.i.d. processes can not be refuted based on spectral measures or covariance structures.

Remark 3. The proof shows that the family of processes with spectral measures absolutely continuous with respect to any fixed measure on $[-1,1]$ is closed under $\bar{d}_{2}$-convergence.

Based on this theorem, we give the characterization of spectral measures of processes that are limits of factor of i.i.d. processes in distribution.

Theorem 4. Fix an infinite vertex-transitive graph G. Suppose that all processes below have marginals with mean 0 and finite second moment. The following are equivalent for a finite (Borel) measure $\mu$ on $\mathbb{R}$.

(i) Its support is contained in the support of the spectral measure of $G$, that is, $\operatorname{supp}(\mu) \subseteq \operatorname{supp}(\nu)$.

(ii) It is the spectral measure of the weak limit of some linear factor of i.i.d. processes.

(iii) It is the spectral measure of the weak limit of some factor of i.i.d. process.

As for the $d$-regular tree, Theorem 5.1 of Backhausz, Szegedy and Virág (2014) gives a description of the pointwise closure of the correlation sequences of factor of i.i.d. processes, which shows the equivalence of $(i)$ and $($ iii $)$ in Theorem 4 after some reformulation.

Remark 5. The family of processes that can be modelled on random $d$ regular graphs in an appropriate sense (see e.g. Backhausz and Szegedy (2014)) is strictly wider than the limit of factor of i.i.d. processes; for example, for large $d$, the independence ratio is twice as large as the proportion of an independent set that can be constructed with factor of i.i.d. (Gamarnik and Sudan 2014, Rahman and Virág|2014). However, 
the possible covariance structures are the same for the two families of invariant random processes, and the results remain valid.

\subsection{Applications.}

Process spectrum. The aim of this section is describing the set of points that can be included in the spectrum of an invariant random process.

Definition 6 (Process spectrum of a graph). Let $G$ be a d-regular vertex transitive graph. Its process spectrum is defined as follows:

$$
\operatorname{psp}(G)=\overline{\bigcup_{X} \operatorname{supp}\left(\mu_{X}\right)} \subseteq[-d, d],
$$

where the union is for all invariant random processes on $G$ with marginals having finite variance. The process spectral radius $\varrho_{p}^{+}(G)$ is defined by $\sup \{x: x \in \operatorname{psp}(G), x<d\}$.

If $d$ is fixed, one can ask whether there are $d$-regular graphs with process spectral radius arbitrarily close to $2 \sqrt{d-1}$.

Theorem 7. Let $\mu$ be a finite measure with $\operatorname{supp}(\mu) \subseteq \operatorname{psp}(G)$. Then there exists an invariant random process $X$ with spectral measure $\mu$.

For the $d$-regular tree it is known that the process spectrum is $[-d, d]$ (Arnaud 1980, 1994, Lasser and Leitner 1990), while the spectrum of the tree is $[-2 \sqrt{d-1}, 2 \sqrt{d-1}]$. In particular, Gaussian wave functions (see the definition below) exist for all $\lambda \in[-d, d]$ (Csóka, Gerencsér, Harangi and Virág 2014, Figà-Talamanca and Nebbia 1991).

We will also prove the following statement about the connection of process spectrum and Kazhdan's property $(T)$ (see e.g. Bekka, de la Harpe and Valette 2008). This also shows that the behavior of process spectral radius and the behavior of spectral radius with respect to tensor product of graphs are different (take the product of two graphs, one with process spectral radius equal to $d$, and the other one less than d).

Proposition 8. Let $H$ be a finitely generated infinite group, and $G$ its Cayley graph with some set of generators. Then the following are equivalent.

(i) H has Kazhdan's property $(T)$.

(ii) The process spectral radius $\varrho_{p}^{+}(G)$ is less than $d$.

$\bar{d}_{2}$-distance and total variation distance of the spectral measures. During the proof of the main theorem, we will show the following inequality and orthogonality. The total variation distance of probability measures will be denoted by $d_{T V}(\cdot, \cdot)$. However, we will use the notion of total 
variation distance not only for probability measures:

$$
d_{T V}\left(\mu_{1}, \mu_{2}\right)=\frac{1}{2} \int|f-g| d \kappa
$$

where $f$ and $g$ are the density functions of $\mu_{1}$ and $\mu_{2}$ with respect to some common dominating measure $\kappa$.

The Hellinger distance $d_{H}$ of measures $\mu, \nu$ is defined by

$$
d_{H}^{2}(\mu, \nu)=\|\mu\|+\|\nu\|-2 \gamma,
$$

where the quantity $\gamma=\int \sqrt{f g} d \kappa$ is the Bhattacharyya coefficient.

Proposition 9. Let $X, Y$ be invariant random processes. Suppose that $\mathbb{E}\left(X_{o}\right)=\mathbb{E}\left(Y_{o}\right)=0$ and the marginals of $X$ have finite second moments.

(a) The following inequality holds:

$$
\begin{aligned}
\bar{d}_{2}^{2}(X, Y) & \geq d_{H}^{2}\left(\mu_{X}, \mu_{Y}\right) \\
& \geq \mathbb{E}\left(X_{o}^{2}\right)+\mathbb{E}\left(Y_{o}^{2}\right)-\sqrt{\left(\mathbb{E}\left(X_{o}^{2}\right)+\mathbb{E}\left(Y_{o}^{2}\right)\right)^{2}-4 d_{T V}^{2}\left(\mu_{X}, \mu_{Y}\right)} \\
& \geq \frac{2 d_{T V}^{2}\left(\mu_{X}, \mu_{Y}\right)}{\mathbb{E}\left(X_{o}^{2}\right)+\mathbb{E}\left(Y_{o}^{2}\right)} .
\end{aligned}
$$

(b) Let $(X, Y)$ be an invariant pair of random processes. Suppose that $\mathbb{E}\left(X_{o}\right)=\mathbb{E}\left(Y_{o}\right)=0$ and the marginals of $X$ and $Y$ have finite second moments. If $\mu_{X}$ and $\mu_{Y}$ are singular measures, then

$$
\mathbb{E}\left(X_{o} Y_{o}\right)=0 .
$$

Linear factor of i.i.d. processes and $\bar{d}_{2}$-metric. Proposition 9 implies that Gaussian factor of i.i.d. processes are closed in the $\bar{d}_{2}$-metric on the $d$-regular tree.

Corollary 10. Let $G=T_{d}$ be the d-regular tree. Suppose that $X^{(n)}$ is a sequence of Gaussian factor of i.i.d. processes such that $X^{(n)} \rightarrow X$ with respect to the $\bar{d}_{2}$-distance as $n \rightarrow \infty$. Then (the distribution of) $X$ is a Gaussian factor of i.i.d. process.

In fact, we will prove this statement for arbitrary vertex-transitive graphs, but for a smaller class of linear factor of i.i.d. processes (see Definition 14 and Subsection 6.3).

Gaussian wave functions. As another application of the inequality in Proposition 9, we will show that Gaussian wave functions are separated from factor of i.i.d. processes with respect to the $\bar{d}_{2}$-distance. A Gaussian wave function with eigenvalue $\lambda$ is an invariant Gaussian process $\left(X_{v}\right)$ satisfying

$$
(A X)_{v}=\lambda X_{v} \text { almost surely for all } v \in V\left(T_{d}\right) .
$$


According to Theorem 4 of Harangi and Virág (2015), such a process exists for all $\lambda$ in the spectrum of the adjacency operator of $G$, but it is not a factor of i.i.d. process if $\lambda$ is the supremum of the spectrum. As for the $d$-regular tree $G=T_{d}$ (with $d \geq 3$ ), Theorem 3 of Csóka, Gerencsér. Harangi and Virág (2015) states that Gaussian wave function $\left(X_{v}\right)$ exists for all $\lambda \in[-d, d]$. Theorem 4 of the same paper says that $\left(X_{v}\right)$ is a weak limit of factor of i.i.d. processes if $\lambda$ is in the spectrum of the tree, i.e. in $[-2 \sqrt{d-1}, 2 \sqrt{d-1}]$, but it is known that it is not a factor of i.i.d. See also Corollary 3.3 of Lyons (2014), which gives a 2-valued example for a process which is weak limit of factor of i.i.d. but not factor of i.i.d.

Proposition 9 will imply the following about wave functions.

Corollary 11. Let $X$ be a Gaussian wave function corresponding to $\lambda \in[-d, d]$ (if it exists) with $\operatorname{Var}\left(X_{o}\right)=1$ and $\mathbb{E}\left(X_{o}\right)=0$.

(a) If $X^{\prime}$ is a Gaussian wave function corresponding to $\lambda^{\prime} \neq \lambda$ with $\mathbb{E}\left(X_{o}^{\prime}\right)=0$ and $\operatorname{Var}\left(X_{o}^{\prime}\right)=1$, then they are orthogonal in every coupling; equivalently, $\bar{d}_{2}\left(X, X^{\prime}\right)=\sqrt{2}$.

(b) If the spectral measure of $G$ is absolutely continuous with respect to the Lebesgue measure, and $Y$ is a limit of factor of i.i.d. processes in the $\bar{d}_{2}$-distance with $\operatorname{Var}\left(Y_{o}\right)=1$, then they are orthogonal in every coupling; equivalently, $\bar{d}_{2}(X, Y)=\sqrt{2}$.

In particular, Gaussian wave functions are never factor of i.i.d. processes. Moreover, the metric space of processes with the $\bar{d}_{2}$-distance is not separable.

Gauss Markov processes. As an application of Theorem 2, we will show the following characterization of factor of i.i.d. Gauss Markov processes on $G=T_{d}$. These are Gaussian processes which have the spatial Markov property. The covariance structure is exponential in this case. That is, for every Markov process there exist $\varrho \in \mathbb{R}$ such that

$$
\operatorname{cov}\left(X_{o}, X_{v}\right)=\varrho^{|v|} \quad\left(v \in V\left(T_{d}\right)\right),
$$

where $|v|$ denotes the distance of $v$ from the root $o$.

Proposition 12. A Gauss Markov process is factor of i.i.d. process if and only if

$$
|\varrho| \leq \frac{1}{\sqrt{d-1}} \quad \text { holds, where } \varrho \text { is defined by equation (2). }
$$


Outline. The paper is built up as follows. In Section 2, we recall the concept of a factor of i.i.d. process and spectral measures. The proofs of Proposition 9, Theorem 2 and Theorem 4 may be found in Sections 3, 4, and 5 respectively. The last section contains the proofs of the applications (process spectrum, $\bar{d}_{2}$-limits of linear factor of i.i.d. processes, Gaussian wave functions, Gaussian free field, Gauss Markov processes).

\section{Preliminaries}

First we recall the definition of factor of i.i.d. processes for vertextransitive graphs. This section is based on Harangi and Virág (2015).

2.1. Invariant random processes and Gaussian processes. Let $G$ be a rooted graph with countable vertex set $V(G)$, edge set $E(G)$ and root $o \in V(G)$. We consider random processes indexed by the vertices of the graph. That is, we assign a random variable $X_{v}$ to each $v \in V(G)$.

We say that a bijection $\Phi: V(G) \rightarrow V(G)$ is an automorphism of $G$ if for all vertices $u$ and $v$ the following holds: $(\Phi(u), \Phi(v)) \in E(G)$ if and only if $(u, v) \in E(G)$. The group of automorphisms of $G$ is denoted by $\operatorname{Aut}(G)$. The graph is vertex transitive if $\operatorname{Aut}(G)$ acts transitively on $V(G)$.

The collection of random variables $\left(X_{v}\right), v \in V(G)$ is an invariant random process on $G$ if for any $\Phi \in \operatorname{Aut}(G)$ the joint distribution of $\left(X_{\Phi(v)}\right)$ is the same as the joint distribution of $\left(X_{v}\right)$.

We say that a collection of random variables $\left(X_{v}\right), v \in V(G)$ is a Gaussian process if their joint distribution is Gaussian (i.e. any finite linear combination of them has Gaussian distribution) and they all have mean 0. An invariant Gaussian process is a Gaussian process that is invariant.

2.2. Factor of i.i.d. processes. Let $G$ be a vertex transitive graph and $\Omega=\mathbb{R}^{V(G)}$. Let $P$ be the product measure on $\Omega$ obtained from the standard normal distribution on $\mathbb{R}$. That is, if we assign independent standard normal random variables $Z_{v}$ to the vertices $v \in V(G)$, then the collection of random variables $\left(Z_{v}\right)$ is a random element of the measure space $(\Omega, P)$.

A factor of i.i.d. process on $G$ will be determined by a function $f \in L^{2}(\Omega, P)$ which is invariant under the root-preserving automorphisms of $G$. Given the independent standard normal random variables $\left(Z_{u}\right)_{u \in V(G)}$, we assign to each vertex $v \in V(G)$ the value of $f$ on $\left(Z_{\Phi(u)}\right)_{u \in V(G)}$ where $\Phi$ is in $\operatorname{Aut}(G)$ taking $v$ to the root. Notice that this does not depend on the choice of $\Phi$, because $f$ is invariant under the root-preserving automorphisms of $G$. We get an invariant random 
process $\left(X_{v}\right)_{v \in V(G)}$ this way. In addition, the only possibility to define the process such that each $X_{u}$ is determined from $\left(Z_{v}\right)$ by a measurable function and they are $\operatorname{Aut}(G)$-equivariant (they commute with the natural action of $\operatorname{Aut}(G))$ is to evaluate a measurable function $f$ at each vertex. See Section 3.2. of Harangi and Virág (2015) for the details. On the other hand, since we will deal with the covariance structure of the process, we need $f$ to be in $L^{2}(\Omega, P)$.

Linear factor of i.i.d. processes. Linear factor of i.i.d. processes will be given by $\ell^{2}$-functions on the vertex set of $G$. More precisely, first we define the $\ell^{2}$-space of the graph by

$$
\ell^{2}(G)=\left\{\alpha: V(G) \rightarrow \mathbb{R} \mid \sum_{v \in V(G)} \alpha(v)^{2}<\infty\right\}
$$

In this space we have the inner product as usual:

$$
\langle\alpha, \beta\rangle_{G}=\sum_{v \in V(G)} \alpha(v) \beta(v) \quad\left(\alpha, \beta \in \ell^{2}(G)\right) .
$$

Definition 13 (Linear factor of i.i.d. process). We say that $X$ is a linear factor of i.i.d. process if its rule $f$ is given by

$$
f(\omega)=\sum_{u \in V(G)} \beta(u) \omega_{u} \quad(\omega \in \Omega)
$$

where $\beta \in \ell^{2}(G)$ and it is invariant under the root-preserving automorphisms of $G$ (that is, $\beta(u)=\beta(\Phi(u)$ ) for all $\Phi \in \operatorname{Aut}(G)$ with $\Phi(o)=o)$.

By Kolmogorov's three-series theorem the sum in the definition is convergent almost surely if and only if $\sum_{u \in V(G)} \beta^{2}(u)<\infty$. See Proposition 3.3. of Harangi and Virág (2015) for more details.

Spherical linear factor of i.i.d. processes. We will also consider a special class of linear factor of i.i.d. processes. In this case the coefficients belong to the $\ell^{2}$-closure of the polynomials of the adjacency operator of $G$. Let the adjacency operator $A: \ell^{2}(G) \rightarrow \ell^{2}(G)$ defined by

$$
(A \beta)(v)=\sum_{(u, v) \in E(G)} \beta(u) \quad\left(v \in G, \beta \in \ell^{2}(G)\right) .
$$

We denote by $\delta_{o} \in \ell^{2}(G)$ the indicator function of the root. For each polynomial $p$ the function $p(A) \delta_{o}$ is a finitely supported function on $V(G)$, hence it is in $\ell^{2}(G)$. Let

$$
\mathcal{L}=\overline{\left\{p(A) \delta_{o}: p \text { is a polynomial }\right\}} \subseteq \ell^{2}(G),
$$

that is, the $\ell^{2}$-closure of the functions given by the polynomials of $A$. 
Definition 14 (Spherical linear factor of i.i.d.). We say that $X$ is a spherical linear factor of i.i.d. process if its rule $f$ is given by

$$
f(\omega)=\sum_{u \in V(G)} \beta(u) \omega_{u} \quad(\omega \in \Omega)
$$

where $\beta \in \mathcal{L}$.

Note that for the regular tree $G=T_{d}$ every finitely supported radial function on $V(G)$ (i.e., the value depends only on the distance from the root) is in the form $p(A) \delta_{o}$ for some polynomial $p$. Hence $\mathcal{L}=\ell^{2}\left(T_{d}\right)$, and every linear factor of i.i.d. process is spherical.

Linear factors. In the sequel, we will also consider finite linear factors of any invariant process $X$. The definition is based on polynomials of $A$, as follows.

Definition 15 (Linear factor of a process $X$ ). Let $X$ be an invariant random process and $p$ a polynomial. By $p(A) X$ we mean the linear factor process defined by

$$
[p(A) X]_{v}=\sum_{w \in V(G)}\left(p(A) \delta_{o}\right)(\Phi(w)) X_{w},
$$

where $\Phi \in \operatorname{Aut}(G)$ is any automorphism taking $v$ to the root $o$.

Since $p(A) \delta_{o}$ is a fixed point of root-preserving automorphisms of $G$, the definition does not depend on the choice of $\Phi$.

\subsection{Spectral measures.}

Spectral measure of the graph. Let $G$ be a vertex-transitive graph with all vertices having degree $d$. Recall that $\delta_{o} \in \ell^{2}(G)$ is the function that is equal to 1 at the root and 0 everywhere else. The operator $A$ is a bounded self-adjoint operator. Therefore there exists a finite measure $\nu$ on $[-d, d]$ such that

$$
\left\langle A^{k} \delta_{o}, \delta_{o}\right\rangle_{G}=\int t^{k} d \nu(t)
$$

holds for every $k \geq 0$. This is called the spectral measure of the graph at the root (see e.g. Bordenave, Sen and Virág (2013), Abért, Thom and Virág (2014) and the references therein). Notice that this is closely related to the return probabilities of the random walk on the graph: the left hand side is the number of returning paths of length $k$ starting from the root.

When $G=T_{d}$ is the infinite $d$-regular tree $(d \geq 3)$, then $\nu$ is the Plancherel (or Kesten-McKay) measure (see e.g. Woess 2000), which 
has density function

$$
h(t)=\left\{\begin{aligned}
\frac{d}{2 \pi} \frac{\sqrt{4(d-1)-t^{2}}}{d^{2}-t^{2}} & t \in[-2 \sqrt{d-1}, 2 \sqrt{d-1}] \\
h(t)=0 & \text { otherwise }
\end{aligned}\right.
$$

with respect to the Lebesgue measure. Here $\varrho=2 \sqrt{d-1}$ is the spectral radius of the tree.

Spectral measure of invariant processes. The spectral measure of invariant random processes will be the spectral measures of the graphings (as bounded self-adjoint operators) associated to them (see also Backhausz et al. (2015)). This concept is as follows.

Let $X$ be an invariant random process with marginals having finite variance. We modify the definition of $\Omega=\mathbb{R}^{V(G)}$ a bit. Namely, we identify two elements if one can be obtained from the other by applying a root-preserving automorphism of $G$. We get $\tilde{\Omega}$ this way. The distribution of $X$ is a probability measure $Q$ on $\tilde{\Omega}$.

We construct a bounded degree graph from $\tilde{\Omega}$ by connecting two elements if and only if one can be obtained from the other one by moving the root to one of its neighbors. Let $\mathcal{E}$ be the edge set of this graph.

Remark 16 . The probability space $(\tilde{\Omega}, Q)$ with edge set $\mathcal{E}$ will form a graphing. See Hatami. Lovász and Szegedy (2014, Section 3) or Backhausz, Szegedy and Virág (2015, Section 2) for the definition and details.

We define the following operator $\mathcal{G}$ acting on $L^{2}(\tilde{\Omega}, Q)$ :

$$
(\mathcal{G} f)(\omega)=\sum_{\left(\omega, \omega^{\prime}\right) \in \mathcal{E}} f\left(\omega^{\prime}\right) \quad\left(f \in L^{2}(\tilde{\Omega}, Q), \omega \in \tilde{\Omega}\right) .
$$

Notice that $\mathcal{G}$ is a bounded self-adjoint operator. Let us denote by $e_{o} \in L^{2}(\tilde{\Omega}, Q)$ the function that assigns to each element of $\tilde{\Omega}$ its value at the root.

According to the spectral theorem, the operator $\mathcal{G}$ has a (finite) spectral measure $\mu_{X}$ at $e_{o}$. That is, $\mu_{X}$ is a finite measure on $\mathbb{R}$ satisfying the following:

$$
\left\langle\mathcal{G}^{k} e_{o}, e_{o}\right\rangle_{Q}=\int t^{k} d \mu_{X}(t) \quad(k \geq 0)
$$

where $\langle\cdot, \cdot\rangle_{Q}$ is the scalar product in $L^{2}(\tilde{\Omega}, Q)$. Since the degrees in $G$ are bounded by $d$, the same holds for the graph constructed above. This implies that the largest eigenvalue (in absolute value) is equal to $d$, and the support of $\mu_{X}$ is contained in the interval $[-d, d]$. 
Definition 17 (Spectral measure of a process). The spectral measure of an invariant random process $X$ (with marginals having finite second moments) is the spectral measure of $\mathcal{G}$ at $e_{o} \in L^{2}(\tilde{\Omega}, Q)$, which is a finite measure on $[-d, d]$. It will be denoted by $\mu_{X}$.

In the sequel, we will use the notation

$$
\langle X, Y\rangle=\mathbb{E}\left(X_{o} \cdot Y_{o}\right)
$$

for any invariant pair of random processes $(X, Y)$ on $G$ which have marginals with finite second moments.

Notice that $X$ is a random element of $\tilde{\Omega}$ with distribution $Q$, the function $e_{o}$ selects the value at the root, and the action of $\mathcal{G}$ corresponds to the linear factor process given by $A$ (recall Definition 15). We obtain

$$
\left\langle\mathcal{G}^{k} e_{o}, e_{o}\right\rangle_{Q}=\mathbb{E}\left(\left[A^{k} X\right]_{o} \cdot X_{o}\right)=\left\langle A^{k} X, X\right\rangle .
$$

We conclude that for every invariant process $X$ its spectral measure $\mu_{X}$ satisfies

$$
\left\langle A^{k} X, X\right\rangle=\int t^{k} d \mu_{X}(t) \quad(k \geq 0), \text { and }
$$

$$
A \text { is self-adjoint: }\left\langle A^{k} X, A^{l} X\right\rangle=\left\langle A^{k+l} X, X\right\rangle \quad(k, l \geq 0) .
$$

Covariance structure. We will define the covariance structure of a process, and we will see how it is related to the spectral measure if the marginals of the process have mean 0 .

Definition 18 (Covariance structure). Let $X$ be an invariant random process with marginals having finite second moments. Its covariance structure, $c_{X}: V(G) \rightarrow \mathbb{R}$ is defined by

$$
c_{X}(v)=\operatorname{cov}\left(X_{o}, X_{v}\right) \quad(v \in V(G)) .
$$

Suppose that $\mathbb{E}\left(X_{o}\right)=0$. The scalar product $\left\langle A^{k} X, X\right\rangle$ is the same as the following: take $X$, start a random walk of length $k$ from the root, calculate the covariance of the values of $X$ at the root and at the endpoint of the random walk, and multiply this by $d^{k}$. On the other hand, by linearity of expectation, we obtain

$$
\left\langle A^{k} X, X\right\rangle=\left\langle A^{k} \delta_{o}, c_{X}\right\rangle_{G} \quad(k \geq 0)
$$

Notice that this is a finite sum. By equation (44) we get

$$
\left\langle A^{k} \delta_{o}, c_{X}\right\rangle_{G}=\int t^{k} d \mu_{X}(t) \quad(k \geq 0),
$$

which shows the connection between the covariance structure and the spectral measure of the process.

Notice that if $X$ is a process that assigns independent random variables to the vertices of $G$, then $c_{X}=\delta_{o}$ and $\mu_{X}=\nu$; that is, the spectral measure of the process is the spectral measure of the graph. 
2.4. Limits of random processes. The $\bar{d}_{2}$-distance of invariant processes (Definition 1) induces the notion of $\bar{d}_{2}$-limits of factor of i.i.d. processes.

We will also deal with distributional convergence. That is, a sequence of invariant random processes converges if the sequence of their distributions on $\Omega=\mathbb{R}^{V(G)}$ converges weakly to some probability measure on $\Omega$. A weak limit of factor of i.i.d. process is an invariant process that can be approximated with factor of i.i.d. processes in distribution. Notice that $\bar{d}_{2}$-convergence implies convergence in distribution.

\section{Proof of Proposition 9}

Before proving Proposition 9, we need a lemma. Recall that $p(A) X$ denotes the linear factor process of $X$ whose coefficients are given by the finitely supported function $p(A) \delta_{o}$ (Definition 15).

Lemma 19 (Isometry). Let $X$ be a random invariant process and $p$ a polynomial. Assume that $\mathbb{E}\left(X_{o}\right)=0$ and the marginals of $X$ have finite second moments. Then we have

$$
\mathbb{E}\left[[p(A) X]_{o}^{2}\right]=\int p^{2} d \mu_{X}
$$

Proof. Using the definition of the spectral measure $\mu_{X}$ (equation (4)) and the fact that $A$ is self-adjoint by equation (5), we get

$$
\int p^{2} d \mu_{X}=\left\langle p^{2}(A) X, X\right\rangle=\langle p(A) X, p(A) X\rangle=\mathbb{E}\left[[p(A) X]_{o}^{2}\right] .
$$

Notice that by the inequality of arithmetic and geometric means, the Bhattacharyya coefficient can be expressed as

$$
2 \gamma=\inf _{\substack{h>0 \\ h, 1 / h \text { integrable }}} \int h d \mu+\int \frac{1}{h} d \nu ;
$$

or, equivalently, using polynomials:

$$
2 \gamma=\lim _{\varepsilon \downarrow 0} \inf _{\substack{p, q \text { polynomials } \\|p q-1|<\varepsilon \text { on supp }(\kappa)}}\left\{\int p^{2} d \mu+\int q^{2} d \nu\right\} .
$$

Proof of Proposition 9. Take any invariant coupling of $X, Y$. Let $\kappa=\mu_{X}+\mu_{Y}$, and $d \mu_{X}=f d \kappa, d \mu_{Y}=g d \kappa$. By Lemma 19] and equation (11), we need to show $\left|\mathbb{E}\left(X_{o} Y_{o}\right)\right| \leq \gamma$. For polynomials $p, q$ as in equation (7), we have

$$
\begin{aligned}
\mathbb{E}\left((p(A) X)_{o}^{2}\right) & +\mathbb{E}\left((q(A) Y)_{o}^{2}\right) \geq 2\left|\mathbb{E}\left(p(A) X_{o} \cdot q(A) Y_{o}\right)\right| \\
& =2\left|\mathbb{E}\left(q(A) p(A) X_{o} \cdot Y_{o}\right)\right|=2\left|\mathbb{E}\left(X_{o} Y_{o}\right)\right|+r,
\end{aligned}
$$

where $|r| \leq \varepsilon \sqrt{\mathbb{E}\left(X_{o}^{2}\right) \mathbb{E}\left(Y_{o}^{2}\right)}$ holds for the error. We conclude by letting $\varepsilon \rightarrow 0$. 
The second inequality is standard, see e.g. Lemma 12.2. in Harsha (2011). We include a proof for completeness. We define

$$
\Delta_{1}=\int|f-g| d \kappa ; \quad s=\int(f+g) d \kappa .
$$

By the Cauchy-Schwarz inequality, we have

$$
\int(\sqrt{f}-\sqrt{g})^{2} d \kappa \int(\sqrt{f}+\sqrt{g})^{2} d \kappa \geq\left[\int|f-g| d \kappa\right]^{2}=\Delta_{1}^{2} .
$$

On the other hand, the definition of $s$ implies that

$$
\int(\sqrt{f}+\sqrt{g})^{2} d \kappa=\int f+g+2 \sqrt{f g} d \kappa=2 s-\int(\sqrt{f}-\sqrt{g})^{2} d \kappa .
$$

Hence for $q=\int(\sqrt{f}-\sqrt{g})^{2} d \kappa$ we obtain $q(2 s-q) \geq \Delta_{1}^{2}$. It follows
that $q \geq s-\sqrt{s^{2}-\Delta_{1}^{2}}$.

The last inequality is straightforward.

As for $(b)$, singularity of $\mu_{X}$ and $\mu_{Y}$ implies that $\int \sqrt{f g} d \eta=0$. Hence the statement follows from the above inequality $\left|\mathbb{E}\left(X_{o} Y_{o}\right)\right| \leq \gamma$.

\section{Proof of Theorem 2}

Lemma 20 (Spectral measures of spherical linear functions). There exists an isometry between $\mathcal{L}$ and $L^{2}(\mathbb{R}, \nu)$ such that the following hold.

(a) For all $\alpha \in \mathcal{L}$ and its image $\hat{\alpha}$ we have

$$
\langle\alpha, \alpha\rangle_{G}=\int \hat{\alpha}^{2}(t) d \nu(t) .
$$

(b) Let $\alpha \in \mathcal{L}$. Then the spectral measure of the spherical linear factor of i.i.d. process given by $\alpha$ has density $\hat{\alpha}^{2}$ with respect to $\nu$.

(c) Let $h \in L^{2}(\mathbb{R}, \nu)$ be a measurable function. Then there exists a spherical linear factor of i.i.d. process $X$ such that $\mu_{X}$ is the measure with density function $h^{2}$ with respect to $\nu$.

Proof. (a) Let $p$ be a polynomial. By equation (3) we have

$$
\left\langle p(A) \delta_{o}, p(A) \delta_{o}\right\rangle_{G}=\int p^{2}(t) d \nu(t) .
$$

It follows that $p(A) \delta_{o} \mapsto p$ is an isometry from the appropriate subspace of $\ell^{2}(G)$ to the set of polynomials in $L^{2}(\mathbb{R}, \nu)$. As usual, this isometry has a unique extension to the $\ell^{2}$-closure; that is, there is an isometry between $\mathcal{L}$ and $L^{2}(\mathbb{R}, \nu)$ (as polynomials form a dense set in the latter space).

(b) Let $\left(p_{n}\right)$ be a sequence of polynomials tending to $\hat{\alpha}$ in $L^{2}(\mathbb{R}, \nu)$. Let $Y$ be an i.i.d. process, and $X$ is the spherical linear factor of i.i.d. process obtained by $\alpha$ from $Y$. The Cauchy-Schwarz inequality implies

$$
\lim _{n \rightarrow \infty}\left\langle A^{k} p_{n}(A) Y, p_{n}(A) Y\right\rangle=\left\langle A^{k} X, X\right\rangle \quad(k \geq 0) .
$$


By equations (3) and (6) this yields

$\lim _{n \rightarrow \infty} \int t^{k} p_{n}^{2}(t) d \nu(t)=\lim _{n \rightarrow \infty}\left\langle A^{k} p_{n}(A) \delta_{o}, p_{n}(A) \delta_{o}\right\rangle_{G}=\left\langle A^{k} X, X\right\rangle(k \geq 0)$.

On the other hand, since $\left(p_{n}\right)$ tends to $\hat{\alpha}$ in $L^{2}(\mathbb{R}, \nu)$, we conclude that

$$
\left\langle A^{k} X, X\right\rangle=\int t^{k} \hat{\alpha}^{2}(t) d \nu(t) \quad(k \geq 0) .
$$

This implies that $d \mu_{X}=\hat{\alpha}^{2} d \nu$, and the proof of part $(b)$ is complete.

(c) Based on the isometry between $\mathcal{L}$ and $L^{2}(\mathbb{R}, \nu)$, let $\alpha \in \mathcal{L}$ be chosen such that $\hat{\alpha}=h$. Let $X$ be the spherical linear function given by $\alpha$. The argument in the proof of $(b)$ implies that the spectral measure of $X$ has density $h^{2}$ with respect to $\nu$.

Lemma 21. Let $X$ be an invariant random process such that $\mathbb{E}\left(X_{o}\right)=$ $0, X$ has marginals with finite second moments, and $c_{X} \in \ell^{2}(G)$. Then $\mu_{X}$ is absolutely continuous with respect to the spectral measure $\nu$ of the graph.

Proof. Suppose first that $c_{X}=p(A) \delta_{o}$ for some polynomial $p$. Then, by self-adjointness and equation (3), we have

$$
\left\langle A^{k} \delta_{o}, c_{X}\right\rangle_{G}=\left\langle A^{k} \delta_{o}, p(A) \delta_{o}\right\rangle_{G}=\int t^{k} p(t) d \nu(t) \quad(k \geq 0) .
$$

By equation (6) we obtain that the measure with density function $p$ will be the spectral measure of $X$. Hence $\mu_{X}$ is absolutely continuous with respect to $\nu$.

Next, we assume that $c_{X} \in \mathcal{L}$. Let $\left(p_{n}\right)$ be a sequence of polynomials converging to $c_{X}$ in $\ell^{2}(G)$. Taking limits in the previous equation and applying Cauchy-Schwarz inequality we obtain that

$$
\begin{aligned}
\left\langle A^{k} \delta_{o}, c_{X}\right\rangle & =\lim _{n \rightarrow \infty}\left\langle A^{k} \delta_{o}, p_{n}(A) \delta_{o}\right\rangle_{G}=\lim _{n \rightarrow \infty} \int t^{k} p_{n}(t) d \nu(t) \\
& =\int t^{k} \widehat{c_{X}} d \nu(t),
\end{aligned}
$$

where $\widehat{c_{X}} \in L^{2}([-d, d], \nu)$ is the image of $c_{X}$ at the isomorphism defined in Lemma 20. Again, this implies that $\mu_{X}$ is absolutely continuous with respect to $\nu$ with density $\widehat{c_{X}}$.

Finally, let $c_{X} \in \ell^{2}(G)$ be arbitrary. By definition, $\mathcal{L}$ is a closed linear subspace in the Hilbert space $\ell^{2}(G)$. Let $\overline{c_{X}} \in \mathcal{L}$ be the projection of $c_{X}$ into this subspace. Since the projection does not change the scalar product with vectors from the subspace, we have

$$
\left\langle A^{k} \delta_{o}, c_{X}\right\rangle_{G}=\left\langle A^{k} \delta_{o}, \overline{c_{X}}\right\rangle_{G} \quad(k \geq 0) .
$$


Therefore the spectral measure of $X$ is the same as the spectral measure corresponding to $\overline{c_{X}}$, which is absolutely continuous with respect to $\nu$.

Proof of Theorem 2. We start with showing that $(i)$ implies (ii). Let $\mu$ be a finite measure, which is absolutely continuous with respect to the spectral measure $\nu$; its density is $g \geq 0$. Since $\sqrt{g} \in L^{2}([-d, d], \nu)$, by part $(c)$ of Lemma 20 there exists a spherical linear factor of i.i.d. process with spectral measure having density $g$ with respect to $\nu$.

$($ ii $) \Rightarrow($ iii $),($ iii $) \Rightarrow($ iv $)$ are trivial.

$(i v) \Rightarrow(v)$ We say that a factor of i.i.d. process is a block factor of i.i.d. if the factor map depends only on the values in a ball of finite radius.

A factor of i.i.d. process can be approximated with block factor of i.i.d. processes in the $\bar{d}_{2}$-distance. See Lyons (2014) for trees, but the proof is similar for vertex-transitive graphs. (To see this, let $f \in L^{2}(\Omega, P)$ be the rule of a factor of i.i.d. process, and let $\mathcal{F}_{r}=$ $\sigma\left\{\omega_{v}:|v| \leq r\right\} \subset \Omega$. By the martingale convergence theorem, the sequence of block factor of i.i.d. processes with rule $\mathbb{E}\left(f \mid \mathcal{F}_{r}\right)$ converges to the factor of i.i.d. process with rule $f$ in the $\bar{d}_{2}$-metric.) Thus, $\bar{d}_{2^{-}}$ limits of factor of i.i.d. processes can also be approximated with block factors. The covariance structure of a block factor of i.i.d. process is finitely supported, hence it is in $\ell^{2}(G)$. Thus every block factor of i.i.d. process has absolutely continuous spectral measure by Lemma 21. This shows that $(i v)$ implies $(v)$.

Finally, we prove that $(v)$ implies $(i)$. Let $\left(X^{(n)}\right)_{n \geq 1}$ be a sequence of invariant random processes such that each $\mu^{(n)}=\mu_{X^{(n)}}$ is absolutely continuous with respect to the spectral measure $\nu$ with density function $g^{(n)}$. Furthermore, assume that $X^{(n)} \rightarrow X$ in the $\bar{d}_{2}$-distance as $n \rightarrow \infty$.

It follows from the Cauchy-Schwarz inequality and the definition of $\bar{d}_{2}$-convergence that $\operatorname{Var}\left(X_{o}^{(n)}\right) \rightarrow \operatorname{Var}\left(X_{o}\right)$ holds as $n \rightarrow \infty$. Therefore Proposition 9 implies that

$$
d\left(\mu^{(n)}, \mu_{X}\right)_{T V} \rightarrow 0 \quad(n \rightarrow \infty) .
$$

We have

$$
d\left(\mu^{(n)}, \mu^{(m)}\right)_{T V}=\frac{1}{2} \int\left|g^{(n)}-g^{(m)}\right| d \nu \quad(n, m \geq 1) .
$$

Equation (8) implies that $\left(g^{(n)}\right)$ is a Cauchy sequence in $L^{1}([-d, d], \nu)$. Due to the completeness of this space, we get that there exists $g \in$ $L^{1}([-d, d], \nu)$ such that

$$
g^{(n)} \rightarrow g \quad \text { in } L^{1}([-d, d], \nu) \quad(n \rightarrow \infty) .
$$

The dominated convergence theorem implies that

$$
\mu^{(n)}=g^{(n)} d \nu \stackrel{T V}{\rightarrow} g d \nu \quad(n \rightarrow \infty)
$$


with respect to the total variation distance (by this notation, we mean the measures having the given density with respect to $\nu$ ). Hence $d \mu_{X}=$ $g d \nu$, and we conclude that $\mu_{X}$ is absolutely continuous with respect to the spectral measure of the graph $G$.

\section{Proof of Theorem 4}

Lemma 22. Let $\left(X^{(n)}\right)$ be a sequence of invariant random processes (with marginals having finite second moments) with covariance structures $\left(c_{n}\right)$ and spectral measures $\left(\mu_{n}\right)$.

(a) Suppose that $X$ is another invariant random process such that

$$
c_{n}(v) \rightarrow c_{X}(v) \text { for all } v \in V(G) \text { as } n \rightarrow \infty \text {. }
$$

Then $\mu_{n} \rightarrow \mu_{X}$ weakly as $n \rightarrow \infty$. In particular, $\mu_{n}$ tends to $\mu$ weakly if $X^{(n)}$ tends to $X$ in distribution and each $X^{(n)}$ is a Gaussian process.

(b) Suppose that $\mu_{n}$ converges weakly to a finite measure $\mu$. Then there exists an invariant random process $X$ with spectral measure $\mu=$ $\mu_{X}$.

Proof. (a) Since every $p(A) \delta_{o}$ has finite support, by equation (3) we obtain that

$$
\int p(t) d \mu_{n}(t)=\left\langle p(A) \delta_{o}, c_{n}\right\rangle_{G} \rightarrow\left\langle p(A) \delta_{o}, c\right\rangle_{G}=\int p(t) d \mu(t)
$$

holds as $n \rightarrow \infty$ for all polynomials $p$. The set of polynomials is dense in the set of continuous functions with respect to the supremum norm, hence $\mu_{n}$ tends to $\mu$ weakly.

Furthermore, in case of Gaussian processes, convergence in distribution implies the pointwise convergence of the covariance structures.

(b) We neglect the degenerate case $\mu([-d, d])=0$. Lemma 19$]$ and the finiteness of $\mu$ implies that $\mathbb{E}\left(\left[X_{o}^{(n)}\right]^{2}\right)$ is bounded. Hence the covariance structures are uniformly bounded by the Cauchy-Schwarz inequality. Since the vertex set of $G$ is countable, we may choose a subsequence $X^{\left(n_{m}\right)}$ such that

$$
c_{n_{m}}(v) \rightarrow c(v) \text { for all } v \in V(G) \text { as } m \rightarrow \infty
$$

for some $c: V(G) \rightarrow \mathbb{R}$. Pointwise convergence preserves the property of being a covariance structure of an invariant process, hence there exists an invariant Gaussian process $X$ with covariance structure $c$. On the other hand, equation (9) and part (a) imply that $\mu_{n_{m}} \rightarrow \mu_{X}$ weakly as $m \rightarrow \infty$. We conclude that $\mu=\mu_{X}$.

Proof of Theorem 4 . (ii) $\Rightarrow$ (iii) is trivial.

$($ iii $) \Rightarrow(i)$ Suppose that the invariant process $X$ is the limit of the factor of i.i.d. processes $\left(X^{(n)}\right)$ in distribution, and $X$ has marginals 
with finite second moments. Denote by $X^{(n, T)}$ the truncation of $X^{(n)}$ at level $T$ :

$$
X_{v}^{(n, T)}=\left\{\begin{array}{ll}
T, & \text { if } X_{v}^{(n)}>T \\
X_{v}^{(n)}, & \text { if } X_{v}^{(n)} \in[-T, T] ; \\
-T, & \text { if } X_{v}^{(n)}<-T ;
\end{array} \quad\left(v \in V\left(T_{d}\right)\right) .\right.
$$

Let $X^{T}$ be the truncated version of $X$.

Since the truncation is a continuous function, $X^{(n, T)} \rightarrow X^{T}$ in distribution as $n \rightarrow \infty$. By boundedness, we have

$$
\operatorname{cov}\left(X_{v}^{(n, T)}, X_{w}^{(n, T)}\right) \rightarrow \operatorname{cov}\left(X_{v}^{T}, X_{w}^{T}\right) \quad(n \rightarrow \infty)
$$

for all $v, w \in V\left(T_{d}\right)$ and $T>0$.

The second moment condition on $X$ implies

$$
\operatorname{cov}\left(X_{v}^{T}, X_{w}^{T}\right) \rightarrow \operatorname{cov}\left(X_{v}, X_{w}\right) \quad(T \rightarrow \infty)
$$

for all $v, w \in V(G)$.

Since $X^{(n)}$ is a factor of i.i.d. process, $X^{(n, T)}$ is also factor of i.i.d. By (10), (11) and a diagonalization argument we obtain that the covariance structure of $X$ is the pointwise limit of covariance structures of factor of i.i.d. processes. (Note that $\operatorname{cov}\left(X_{v}^{(n)}, X_{w}^{(n)}\right)$ may not converge to $\operatorname{cov}\left(X_{v}, X_{w}\right)$.)

To summarize, we can find a sequence of factor of i.i.d. covariance structures $\left(c_{m}\right)$ such that $c_{m}(v) \rightarrow c_{X}(v)$ for all $v \in V(G)$ as $m \rightarrow \infty$. Part (a) of Lemma 22 implies that $\mu_{m}$ tends to $\mu$ weakly. By Theorem 2 every $\mu_{m}$ is absolutely continuous with respect to $\nu$. Therefore the support of $\mu$ is contained in $\operatorname{supp}(\nu)$.

$(i) \Rightarrow(i i)$ Let $\mu$ be a finite measure such that $\operatorname{supp}(\mu) \subseteq \operatorname{supp}(\nu)$. There exist a sequence of finite measures $\left(\mu_{n}\right)$ such that it converges weakly to $\mu$ and every $\mu_{n}$ is absolutely continuous with respect to $\nu$.

On the other hand, Theorem 2 implies that for every $\mu_{n}$ there exists a spherical linear factor of i.i.d process $X^{(n)}$ whose spectral measure is $\mu_{n}$. It follows from part (b) of Lemma 22 that $\mu$ is the spectral measure of some Gaussian process $X$. In addition, the proof of the lemma shows that the covariance structures of a subsequence of $X^{(n)}$ converge pointwise to the covariance structure of $X$. Since every $X^{(n)}$ is spherical, it is also Gaussian. In case of Gaussian processes the pointwise convergence of covariance structures implies convergence in distribution. Therefore some subsequence of $\left(X^{(n)}\right)$ converges to $X$ in distribution, which concludes the proof.

Remark 23. Notice that linear factor of i.i.d. processes are always Gaussian. Therefore for an absolutely continuous measure we can find a Gaussian factor of i.i.d. process with this spectral measure. Similarly, for measures supported on $\operatorname{supp}(\nu)$, the appropriate process is the weak limit of Gaussian factor of i.i.d. processes as well. 


\section{Applications}

\subsection{Process spectrum.}

Proof of Theorem 7. First we prove the statement for atomic measures. Suppose that $x \in \operatorname{psp}(G)$, and $\mu$ is an atom at $x$. Given $\varepsilon>0$, there exists a process $X^{\varepsilon}$ such that $\mu_{X^{\varepsilon}}([x-\varepsilon, x+\varepsilon])>0$. It follows that there exists a polynomial $p_{\varepsilon}$ such that the following hold for the measure $d \mu_{\varepsilon}^{*}=p_{\varepsilon} d \mu_{X^{\varepsilon}}$ (i.e. the measure $\mu_{\varepsilon}^{*}$ that has density function $p_{\varepsilon}$ with respect to $\left.\mu_{X^{\varepsilon}}\right)$ :

$$
\mu_{\varepsilon}^{*}(\mathbb{R} \backslash[x-\varepsilon, x+\varepsilon])<\varepsilon ; \quad \mu(x)-\varepsilon \leq \mu_{\varepsilon}^{*}([x-\varepsilon, x+\varepsilon]) \leq \mu(x)+\varepsilon .
$$

Notice that $\mu_{\varepsilon}^{*}$ tends to $\mu$ weakly as $\varepsilon \rightarrow 0$. Equation (44) implies that the process $p_{\varepsilon}\left(X^{\varepsilon}\right)$, which is a finite linear factor of $X^{\varepsilon}$ by Definition 15, has spectral measure $\mu_{\varepsilon}^{*}$. Putting this together with Lemma 22 (b), we conclude that $\mu$ is the spectral measure of some invariant random process.

Further on, if the support of $\mu$ consists of finitely many atoms, then one can take the sum of independent copies of invariant random processes constructed for atomic measures, multiplied by appropriate constants. The covariance structure is additive due to independence, which shows by equation (6) that the spectral measure is $\mu$.

Finally, if we have an arbitrary finite measure $\mu$ whose support is contained in $\operatorname{psp}(G)$, then it can be approximated weakly with measures $\mu_{n}$, where each $\mu_{n}$ is supported on finitely many atoms. For every $\mu_{n}$ we already have a process $X^{(n)}$ with spectral measure $\mu_{n}$. We finish the proof by applying part $(b)$ of Lemma 22 .

Proof of Proposition 8 . (ii) $\Rightarrow($ i) We will use the next formulation of Kazhdan's property: every sequence of positive definite functions on $G$ that converges to 1 pointwise (i.e. on compact subsets) converges to 1 uniformly on $G$.

Let $X$ be the following process: constant 1 on all vertices with probability $1 / 2$, and constant -1 with probability $1 / 2$. Then $c_{X}(v)=1$ for all $v \in V(G)$, and, by equation (6) it follows that $\mu_{X}=\delta_{d}$ is an atomic measure at $d$.

Let $c_{n}: V(G) \rightarrow \mathbb{R}$ be a sequence of positive definite functions converging pointwise to 1 . We can find a sequence of invariant processes $\left(X^{(n)}\right)$ such that $X^{(n)}$ has covariance structure $c_{n}$. By Lemma $22(a)$ we obtain that $\mu_{X^{(n)}} \rightarrow \mu_{X}$ weakly. It follows from (ii) and the fact $\mu_{X}=\delta_{d}$ that

$$
\mu_{X^{(n)}}=\left(1-\varepsilon_{n}\right) \delta_{d}+\mu_{n}^{\prime}
$$

where $\varepsilon_{n} \rightarrow 0$, and $\left(\mu_{n}^{\prime}\right)$ is a sequence of measures such that $\mu_{n}^{\prime}([-d, d])$ tends to 0 as $n \rightarrow \infty$, and $\mu_{n}^{\prime}$ is $\operatorname{supported~on~} \operatorname{psp}(G)$. 
Since $\mu_{X^{(n)}}$ is the spectral measure corresponding to $X^{(n)}$, we get that $\mu_{n}^{\prime}$ is the spectral measure corresponding to $c_{n}-(1-\varepsilon)$. That is, we have

$$
\left\langle A^{k}\left(c_{n}-(1-\varepsilon)\right), \delta_{o}\right\rangle=\int t^{k} d \mu_{n}^{\prime} \quad(k=0,1, \ldots) .
$$

It follows that $\left(c_{n}-(1-\varepsilon)\right)$ is positive definite for each $n$, and hence it is a covariance structure of an invariant process. The fact $\mu_{n}^{\prime}([-d, d]) \rightarrow 0$ implies that $\left(c_{n}-(1-\varepsilon)\right)(0)$ tends to 0. By the Cauchy-Schwarz inequality we obtain that $c_{n}-(1-\varepsilon)$ converges to 0 uniformly. Hence $c_{n}$ converges to constant 1 uniformly, and $G$ has Kazhdan's property $(T)$.

(i) $\Rightarrow($ ii $)$ Let $X$ be the constant 1 process. Its spectral measure is an atomic measure at $d$, hence $d$ is always in the process spectrum. Suppose (for contradiction) that $d$ is not an isolated point. Choose a sequence of numbers $\left(a_{n}\right)$ with limit $d$ such that $a_{n} \in \operatorname{psp}(G)$ for all $n$. Theorem 7 implies that we can find a sequence of invariant processes $X^{(n)}$ such that the spectral measure of $X^{(n)}$ is an atomic measure at $a_{n}$ for each $n$. It follows that $\mu_{X^{(n)}} \rightarrow \mu_{X}$ weakly as $n \rightarrow \infty$. Similarly to the proof of Lemma 22, we can assume that the covariance structures of $X^{(n)}$ converge pointwise to $c_{X}$, by choosing an appropriate subsequence. By the characterization of Kazhdan's property using positive semidefinite functions (covariance structures are positive semidefinite), we get that $c_{X^{(n)}} \rightarrow c_{X} \equiv 1$ uniformly on $V(G)$.

On the other hand, if $H$ is infinite and has Kazhdan's property $(T)$, then it can not be amenable (see e.g. Bekka, de la Harpe and Valette 2008). Therefore its spectral radius is strictly less than $d$. Therefore the covariance of $X_{o}$ and $X_{v}$, where $v$ is the endpoint of a random walk of length $k$, decays exponentially as a function of $k$. This contradicts the uniform convergence of correlation structures above. Hence the process spectral radius is less than $d$.

\subsection{Gaussian wave functions.}

Proof of Corollary 11. We check that $\mu_{X}$ is the Dirac measure which puts an atom of weight 1 at $\lambda$ :

$$
\left\langle A^{k} X, X\right\rangle=\lambda^{k}\langle X, X\rangle=\lambda^{k} \operatorname{Var}\left(X_{o}\right)=\lambda^{k}=\int t^{k} d \mu_{X}(t) .
$$

Different Dirac measures are singular measures. On the other hand, the measure $\mu_{Y}$ is absolutely continuous with respect to the spectral measure of the graph by Theorem 2. Hence it is absolutely continuous with respect to the Lebesgue measure in case $(b)$, and again, it is singular to the Dirac measure. Therefore both parts of the statement follow from Proposition 9 (b). 
6.3. Linear factor of i.i.d. processes and $\bar{d}_{2}$-convergence. First we prove the following statement about spherical linear processes.

Proposition 24. Suppose that $X^{(n)}$ is a sequence of spherical linear factor of i.i.d. processes such that $X^{(n)} \rightarrow X$ with respect to the $\bar{d}_{2^{-}}$ distance as $n \rightarrow \infty$. Then (the distribution of) $X$ is a spherical linear factor of i.i.d. process.

Proof. Let $\alpha_{n} \in \mathcal{L}$ be the function which defines $X^{(n)}$. Lemma 20 implies $\mu_{X^{(n)}}={\widehat{\alpha_{n}}}^{2} d \nu$. On the other hand, due to the $\bar{d}_{2}$-convergence of $X^{(n)}$ and part $(a)$ of Proposition 9, we get that $\mu_{X^{(n)}}$ is a Cauchy sequence in total variation distance. Therefore, since $L^{1}([-d, d], \nu)$ is complete, we can find a function which is the limit of the sequence $\left({\widehat{\alpha_{n}}}^{2}\right)$. It has to be nonnegative, hence there exists $\hat{\alpha} \in L^{2}([-d, d], \nu)$ such that $\hat{\alpha} \geq 0$ and

$$
\int\left|{\widehat{\alpha_{n}}}^{2}-\hat{\alpha}^{2}\right| d \nu \rightarrow 0 \quad(n \rightarrow \infty) .
$$

Using the inequality $(a-b)^{2} \leq\left|a^{2}-b^{2}\right|$ for $a, b>0$, this yields

$$
\int\left(\widehat{\alpha_{n}}-\hat{\alpha}\right)^{2} d \nu \rightarrow 0 \quad(n \rightarrow \infty) \text {. }
$$

Let $\alpha \in \mathcal{L}$ be the function corresponding to $\alpha$ according to part $(a)$ of Lemma 20. Let $X^{\alpha}$ be the spherical linear factor of i.i.d. process given by $\alpha$. By applying $\alpha_{n}$ and $\alpha$ on the same i.i.d. process (which defines the coupling), and using Lemma 20 (b), we have

$$
\int\left(\widehat{\alpha_{n}}-\hat{\alpha}\right)^{2} d \nu=\mathbb{E}\left[\left(X_{o}^{(n)}-X_{o}^{\alpha}\right)^{2}\right]
$$

Hence $X^{(n)}$ converges to $X^{\alpha}$ in the $\bar{d}_{2^{2}}$-distance. Convergence in $\bar{d}_{2^{-}}$ distance implies convergence in distribution, and the limit is unique. We conclude that the distribution of $X$ is equal to the distribution of $X^{\alpha}$, which is a spherical linear factor of i.i.d. process.

Proof of Corollary 10. Invariant Gaussian processes are determined by their covariance structure. Hence every Gaussian factor of i.i.d. process is a linear factor of i.i.d. process by Theorem 2 ,

On the other hand, every linear factor of i.i.d. process is spherical on the $d$-regular tree, because every finitely supported radial function is a polynomial of $A$. Proposition 24 implies that the limit of the sequence is a linear factor of i.i.d. process. Hence it is Gaussian.

6.4. Gaussian free field. We recall the definition of an analogue of the Gaussian free field on transient graphs, and we will show that all these random processes are factor of i.i.d. processes (see also Exercise 10.31. in Lyons and Peres (2014)). 
Definition 25 (Gaussian free field). Let $G$ be a transient and vertextransitive graph. An invariant Gaussian random process is a Gaussian free field if its covariance structure is the Green function:

$$
\sum_{k=0}^{\infty}\left(\frac{A}{d}\right)^{k}
$$

Notice that our assumption that $G$ is transient implies that this series is convergent, because the number of visits of a random walk starting from the root is finite almost surely.

Proposition 26. A Gaussian free field is a linear factor of i.i.d. process.

Proof. Consider the function defined by

$$
\sum_{k=0}^{\infty}\left(\frac{x}{d}\right)^{k}=\frac{d}{d-x} \quad(x \in(-d, d)) .
$$

The transitivity of $G$ implies that this function is in $L^{1}([-d, d], \nu)$, where $\nu$ is the spectral measure of $G$. It follows from the definition of the Gaussian free field that this is the density function of its spectral measure. Theorem 2 and the fact that Gaussian processes are determined by their covariance structures imply the statement.

6.5. Gaussian Markov processes on the tree. We will need the following family of polynomials, which plays an important role in understanding the radial functions on the regular tree. Let $\left(r_{n}\right)$ be the unique sequence of polynomials satisfying the following recurrence equations:

$$
\begin{aligned}
r_{0}(x) & =1 \\
x r_{0}(x) & =r_{1}(x) \\
x r_{n}(x) & =(d-1) r_{n-1}(x)+r_{n+1}(x) \quad(n \geq 1) .
\end{aligned}
$$

The polynomial $r_{n}$ has degree $n$. These are sometimes called Dunau polynomials, and they are closely related to Chebyshev polynomials of the second kind (see e.g. Alon, Benjamini, Lubetzky and Sodin 2007, Backhausz, Szegedy and Virág 2014, Arnaud and Letac 1984, FigàTalamanca and Nebbia 1991). On the other hand, they are orthogonal with respect to the Plancherel measure. Moreover, the following can be proved by induction: $r_{0}(A) \delta_{o}=\delta_{o}$, and for $n \geq 1$ we have

$$
\left[r_{n}(A) \delta_{0}\right](v)= \begin{cases}1, & \text { if }|v|=n ; \\ 0, & \text { otherwise }\end{cases}
$$

This implies that all radial functions on the tree are given by limits of polynomials of $A$. 
Proof of Proposition 12 . Let $c_{\varrho}: V\left(T_{d}\right) \rightarrow \mathbb{R}$ be defined by $c_{\varrho}(v)=\varrho^{|v|}$. By equation (2), this is the covariance structure of the Gaussian Markov process. First we decide whether $c_{\varrho}$ is in $\ell^{2}\left(T_{d}\right)$ :

$$
1+\sum_{k=1}^{\infty} d(d-1)^{k-1} \varrho^{2 k}<\infty \Leftrightarrow|\varrho|<\frac{1}{\sqrt{d-1}} .
$$

Hence in the case $|\varrho|<\frac{1}{\sqrt{d-1}}$ Lemma 21 applies. However, we compute this density function for $|\varrho|<\frac{1}{\sqrt{d-1}}$, in order to deal with the case $|\varrho|=\frac{1}{\sqrt{d-1}}$. Equations (2) and (13) imply that

$$
c_{\varrho}=\sum_{k=0} \varrho^{k} r_{k}(A) \delta_{o}
$$

Based on the proof of Lemma21, we obtain that the density function (with respect to the spectral measure of the tree) of the spectral measure of the Gaussian Markov process with parameter $\varrho$ is the following:

$$
f_{\varrho}(x)=\sum_{k=0}^{\infty} \varrho^{k} r_{k}(x) .
$$

To compute this sum, let

$$
g(x, y)=\sum_{k=1}^{\infty} r_{k}(x) y^{k} .
$$

Then by recurrence equation (12) we have

$$
\begin{aligned}
x g(x, y) & =(d-1) \sum_{k=1}^{\infty} r_{k-1}(x) y^{k}+\sum_{k=1}^{\infty} r_{k+1}(x) y^{k} \\
& =y(d-1) \sum_{k=0}^{\infty} r_{k}(x) y^{k}+\frac{1}{y} \sum_{k=2}^{\infty} r_{k}(x) y^{k} \\
& =y(d-1) r_{k}(0)+y(d-1) g(x, y)+\frac{1}{y}\left[g(x, y)-r_{1}(x) y\right] \\
& =y(d-1)+y(d-1) g(x, y)+\frac{1}{y} g(x, y)-x
\end{aligned}
$$

This yields

$$
g(x, y)=\frac{x y-y^{2}(d-1)}{1+y^{2}(d-1)-x y} .
$$

Hence the density function of $\mu$ is the following:

$$
f_{\varrho}(x)=g(x, \varrho)+1=\frac{1}{1+\varrho^{2}(d-1)-x \varrho} \quad\left(|\varrho|<\frac{1}{\sqrt{d-1}}\right) .
$$


For $\varrho=\frac{1}{\sqrt{d-1}}$ the covariance structure is not in $\ell^{2}\left(T_{d}\right)$. However, the calculation above works and we get

$$
f(x)=\sum_{k=0}^{\infty} r_{k}(x)(d-1)^{-k / 2}=\frac{1}{2-\frac{x}{\sqrt{d-1}}} .
$$

This has a singularity only at the endpoint of the Plancherel interval, namely, at $2 \sqrt{d-1}$. Since the density function of the Plancherel measure behaves like $\sqrt{x}$ at the endpoints of its support, this function is still integrable with respect to the Plancherel measure: $f \in L^{1}([-d, d], \nu)$. Therefore this is the density function of the spectral measure of the Gauss Markov process with respect to $\nu$. Hence $c_{\varrho}$ is the covariance structure of a factor of i.i.d. process if $|\varrho| \leq 1 / \sqrt{d-1}$. Similar argument works for $\varrho=-1 / \sqrt{d-1}$.) For Gauss Markov processes this implies that the process is linear factor of i.i.d. itself, according to Theorem 2.

As for the other direction, we refer to Backhausz, Szegedy and Virág (2015): from that result it follows immediately that covariance structure with larger absolute value of $\varrho$ can not be factor of i.i.d.

6.6. Branching Markov chains. We can also examine the covariance structures of branching Markov chains on the $d$-regular tree. Fix a reversible Markov chain with finite state space $S$ and transition matrix $M$. Choose the state of the root $o$ uniformly at random. Then the Markov chain spreads out: the neighbors of the root get their states given the state of the root and according to the transition probabilities given by $M$. The transitions are conditionally independent given the state of the root. This is continued to get the states of the neighbors of the neighbors of the root, and so on. This will be an invariant random process on $T_{d}$. One can get the Potts and the Ising model as particular cases (see e.g. Evans, Kenyon, Peres and Schulman 2000, Sly 2011).

Let $\varphi$ be eigenvector of $M$ corresponding to the largest eigenvalue. Then the correlation of $\varphi\left(X_{o}\right)$ and $\varphi\left(X_{v}\right)$ is $\varrho^{|v|}$, where $\varrho$ is the spectral radius of the transition matrix $M$. Therefore the calculation above implies that this covariance structure is a factor of i.i.d. covariance structure if and only if $|\varrho| \leq 1 / \sqrt{d-1}$. However, this does not imply that the process itself is factor of i.i.d. in this case.

A particular case is the Ising model, where $S=\{-1,1\}$ and

$$
M=\left(\begin{array}{ll}
\frac{1+\varrho}{2} & \frac{1-\varrho}{2} \\
\frac{1-\varrho}{2} & \frac{1+\varrho}{2}
\end{array}\right) .
$$

It is known that the process is itself a factor of i.i.d. if $|\varrho| \leq \frac{1}{d-1}$, see e.g. Lyons (2014). It is open whether the Ising model itself is factor of i.i.d. in the case $1 /(d-1)<|\varrho| \leq 1 / \sqrt{d-1}$. 
6.7. Open questions. We finish the paper with some open questions.

(1) Is there a spectral description of the structure determined by the expectation of the product of the random variables at more than two vertices? In this case not just the distance matters, the configuration of the vertices has to be fixed.

(2) Is there a spectral description of the moments of the random variables at the vertices? That is, we assign $X_{v}, X_{v}^{2}, \ldots, X_{v}^{k}$ to each vertex instead of $X_{v}$, and we can take covariance matrices of the vectors assign to a pair of vertices.

Acknowledgement. The authors are especially grateful to Russell Lyons for useful comments that led to a shorter proof of Proposition 9. The authors thank Péter Csikvári, Viktor Harangi and Mustazee Rahman for reading previous versions of the manuscript and providing valuable suggestions, Gérard Letac for references, Gábor Pete for the question on the covariance structure of $\bar{d}_{2}$-limits of factor of i.i.d. processes, and Balázs Szegedy for useful discussions. The first author was partially supported by the National Research, Development and Innovation Office (NKFIH, grant no. 109684), and by the MTA Rényi Institute Lendület Limits of Structures Research Group. The second author was supported by Marie Skłodowska-Curie actions "Spectra", by the NSERC Discovery Accelerator Supplements Program and by the MTA Rényi "Lendület" Groups and Graphs Research Group.

\section{REFERENCES}

Arnaud, J.-P. (1980). Fonctions sphériques et fonctions définies positives sur l'arbre homogène, C. R. Acad. Sci. Paris Sér. A-B 290(2): A99-A101.

Arnaud, J.-P. (1994). Stationary processes indexed by a homogeneous tree, Ann. Probab. 22(1): 195-218.

Backhausz, Á. and Szegedy, B. (2014). On large girth regular graphs and random processes on trees, preprint. (arXiv:1406.4420 [math.PR]).

Backhausz, A., Szegedy, B. and Virág, B. (2015). Ramanujan graphings and correlation decay in local algorithms, Random Structures Algorithms 47(3): 424-435.

Bekka, B., de la Harpe, P. and Valette, A. (2008). Kazhdan's property (T), Vol. 11 of New Mathematical Monographs, Cambridge University Press, Cambridge.

Bowen, L. (2010). The ergodic theory of free group actions: entropy and the $f$-invariant, Groups Geom. Dyn. 4(3): 419-432.

Cartier, P. (1973). Harmonic analysis on trees, Harmonic analysis on homogeneous spaces (Proc. Sympos. Pure Math., Vol. XXVI, 
Williams Coll., Williamstown, Mass., 1972), Amer. Math. Soc., Providence, R.I., pp. 419-424.

Csóka, E., Gerencsér, B., Harangi, V. and Virág, B. (2015). Invariant Gaussian processes and independent sets on regular graphs of large girth, Random Structures Algorithms 47(2): 284-303.

Csóka, E. and Lippner, G. (2012). Invariant random matchings in Cayley graphs, preprint. (arXiv:1211.2374 [math.CO]).

Elek, G. and Lippner, G. (2010). Borel oracles. An analytical approach to constant-time algorithms, Proc. Amer. Math. Soc. 138(8): 29392947.

Evans, W., Kenyon, C., Peres, Y. and Schulman, L. J. (2000). Broadcasting on trees and the Ising model, Ann. Appl. Probab. 10(2): 410433.

Figà-Talamanca, A. and Picardello, M. A. (1982). Spherical functions and harmonic analysis on free groups, J. Funct. Anal. 47(3): 281304.

Gamarnik, D. and Sudan, M. (2014). Limits of local algorithms over sparse random graphs, Proceedings of the 5-th Innovations in Theoretical Computer Science conference, ACM Special Interest Group on Algorithms and Computation Theory .

Harangi, V. and Virág, B. (2015). Independence ratio and random eigenvectors in transitive graphs, Ann. Probab. 43(5): 2810-2840.

Harsha, P. (2011). Communication complexity lecture notes.

URL: $\quad$ http://www.tcs.tifr.res.in/ prahladh/teaching/201112/comm/lectures/l12.pdf

Hatami, H., Lovász, L. and Szegedy, B. (2014). Limits of locallyglobally convergent graph sequences, Geom. Funct. Anal. 24(1): 269296.

Lasser, R. and Leitner, M. (1990). On the estimation of the mean of weakly stationary and polynomial weakly stationary sequences, $J$. Multivariate Anal. 35(1): 31-47.

Lyons, R. (2014). Factors of iid on trees, Combin. Probab. Comput., to appear. (arXiv:1401.4197 [math.DS]).

Lyons, R. and Nazarov, F. (2011). Perfect matchings as IID factors on non-amenable groups, European J. Combin. 32(7): 1115-1125.

Lyons, R. and Peres, Y. (2014). Probability on trees and networks, in preparation.

URL: http://mypage.iu.edu/ ${ }^{\sim}$ rdlyons/

Rahman, M. and Virág, B. (2014). Local algorithms for independent sets are half-optimal, preprint. (arXiv:1402.0485 [math.PR]).

Sly, A. (2011). Reconstruction for the Potts model, Ann. Probab. 39(4): 1365-1406.

Woess, W. (2000). Random walks on infinite graphs and groups, Vol. 138 of Cambridge Tracts in Mathematics, Cambridge University Press, Cambridge. 
Ágnes Backhausz. MTA Alfréd Rényi Institute of Mathematics and Eötvös Loránd University; Budapest, Hungary.

E-mail address: backhausz.agnes@renyi.mta.hu

BÁlint VIRÁG. Department of Mathematics, University of Toronto, Canada and MTA Alfréd Rényi Institute of Mathematics, Budapest, Hungary.

E-mail address: balint@math.toronto.edu 